Article

\title{
A global seamless DEM based on multi-source data fusion (GSDEM-30): Product generation and evaluation
}

\author{
Linwei Yue ${ }^{1}$, Huanfeng Shen ${ }^{2, *}$ Qiangqiang Yuan ${ }^{3}$, Lu Liu ${ }^{2}$ and Liangpei Zhang 4 \\ 1 Faculty of Information Engineering, China University of Geosciences, Wuhan, Hubei, 430074, China; \\ yuelw@cug.edu.cn \\ 2 School of Resource and Environmental Sciences, Wuhan University, Wuhan, Hubei, 430079, China; \\ shenhf@whu.edu.cn, liulu@whu.edu.cn \\ 3 School of Geodesy and Geomatics, Wuhan University, Wuhan, Hubei, 430079, China; yuanqq@whu.edu.cn \\ 4 State Key Laboratory of Information Engineering, Survey Mapping and Remote Sensing, Wuhan \\ University, Wuhan, Hubei, 430079, China; zlp62@whu.edu.cn \\ * Correspondence: shenhf@whu.edu.cn;
}

\begin{abstract}
The quality of digital elevation models (DEMs) is inevitably affected by the limitations of the imaging modes and the generation methods. One effective way to solve this problem is to merge the available datasets through data fusion. In this paper, a fusion-based global DEM dataset $\left(82^{\circ} \mathrm{S}-82^{\circ} \mathrm{N}\right)$ is introduced, which we refer to as GSDEM-30. This is a $30-\mathrm{m}$ DEM mainly reconstructed from the unfilled SRTM1, AW3D30, and ASTER GDEM v2 datasets combining the multi-source and multi-scale fusion techniques. A comprehensive evaluation of the GSDEM-30 data, as well as the 30-m ASTER GDEM v2 and AW3D30 DEM, was presented. Global ICESat GLAS data and the local National Elevation Dataset (NED) were used as the reference for the vertical accuracy validation, while GlobeLand30 was introduced for the landscape analysis. Furthermore, we employed the maximum slope approach to detect the potential artefacts in the DEMs. The results show that the GDEM data are seriously affected by noise and artefacts. With the advantage of the multiple datasets and the refined post-processing, the GSDEM-30 are contaminated with fewer anomalies than both ASTER GDEM and AW3D30. The fusion techniques used can also be applied to the reconstruction of other fused DEM datasets.
\end{abstract}

Keywords: Digital elevation models; multi-source fusion; multi-scale fusion; global evaluation; accuracy validation.

\section{Introduction}

Digital elevation models (DEMs) provide topographic information of the terrain surface, which is a significant data source in the related geospatial applications [1]]. With the rapid development of remote sensing and photogrammetric techniques, there has been a variety of global-scale DEM products derived from satellite observations. The most important datasets include the Shuttle Radar Topography Mission (SRTM) DEMs [2], the Advanced Spaceborne Thermal Emission and Reflection Radiometer (ASTER) Global Digital Elevation Model (GDEM) [3] , the recently developed Advanced Land Observing Satellite (ALOS) World 3D (AW3D) DEM [ $\underline{4}$, and the TanDEM-X DEM [ $\underline{]}$. Unfortunately, the quality of the individual DEM datasets is inevitably affected by the imaging techniques and the generation methods [ $[\underline{6}$. For example, optical data are easily contaminated with anomalies, mainly due to cloud and cloud shadow [7]. Compared with optical observations, radar imaging can provide all-day and all-weather observations. However, it is more sensitive to the terrain slope, and thus voids are common in the radar DEMs with rugged topography []. Furthermore, in the snow- or desert-covered areas with indistinctive texture, both the optical and radar results are often of low quality [9]. Besides, the DEM extraction methods from the 
interferometric signal and stereo images, as well as the post-processing methods, will affect the data accuracy [1ㅇ].

Consequently, the tradeoff between the spatial resolution, spatial coverage, and vertical accuracy means that the currently available datasets do not meet the demands for the large-scale geospatial applications [11]. On the one hand, more advanced imaging and processing technologies will be applied to Earth observation [12], and new products with a higher resolution and fine-edited quality are being generated and released. However, new data generation is not only highly cost and time-consuming, but is also inevitably limited by the imaging techniques [13]. Moreover, the editing of raw DEM data only using the single-source information, e.g. the noise filtering and voids interpolation methods, might fail in the cases where local data quality are poor [14-18]. Thus, considering the auxiliary information among the multi-source datasets, merging the currently available datasets through multi-source data fusion is a possible way to solve this problem. Different fusion techniques were developed to solve the quality issues of DEM data, such as voids, anomalies and different sources of noise $[\underline{14}, \underline{19-24}]$. Based on the fusion methods, great efforts have been made to generate high-quality DEM products by merging the existing datasets $[\underline{19}, \underline{21}, \underline{25}]$.

In this paper, we introduce a fused DEM dataset $\left(82^{\circ} \mathrm{S}-82^{\circ} \mathrm{N}\right)$ mainly reconstructed from the 30-m unfilled SRTM1, AW3D30, ASTER GDEM v2 and ICESat GLAS data, which we refer to as GSDEM-30. The goal is to generate a global seamless DEM using the multi-source accuracy enhancement method and the regularized multi-scale fusion algorithm $[\underline{22}, \underline{26}]$. Considering the global data characteristics, the artificial neural network (ANN) based accuracy enhancement and void filling method were used for the void SRTM tiles between $50^{\circ} \mathrm{S}-50^{\circ} \mathrm{N}$, while the multi-scale fusion algorithm was applied to the SRTM tiles sub-sampled to a 2-arc-second resolution along the longitude within $50-60^{\circ} \mathrm{N}$ and $50^{\circ}-60^{\circ} \mathrm{S}$. Moreover, this paper gives a comprehensive evaluation of ASTER GDEM v2, AW3D30 and GSDEM-30. In the experiments, the globally distributed ICESat GLAS data and a local high-accuracy DEM were used as the reference for the vertical accuracy evaluation. Furthermore, the error distribution with different landforms and land covers was considered in the statistics. In addition, the error propagation to the derived topographic slope was analyzed using the non-reference maximum slope approach (MSA) [27].

\section{Review of the open-source global DEMs}

\subsection{SRTM DEM}

The SRTM mission in February, 2000 continued for 11 days, and recorded a "snapshot" of the terrain surface over $80 \%$ of the globe $\left(56^{\circ} \mathrm{S}-60^{\circ} \mathrm{N}\right)$ by single-pass interferometry [르] . The original SRTM DEM was processed from raw C-band radar images at NASA's Jet Propulsion Laboratory (JPL) with a $\sim 30$-m resolution referenced to the datum WGS84/EGM96. The following SRTM v2.1 and v4.1 were resampled at 3 arc-seconds ( $\sim 90 \mathrm{~m}$ ) outside the United States for public release, with further processing of water-body boundaries, spike and well removal, and void reduction [르]. The most commonly used version is SRTM3 v4.1 with data voids filled [25], with the vertical accuracy reported to be $\sim 16 \mathrm{~m}$ at the $90 \%$ confidence level [30]. However, the void filling results are rough and the accuracy is unstable [22]. From 2014, the global SRTM 1-arc-second data (SRTM1) can be accessed with a higher resolution and better quality, but some tiles still contained voids. Voids filling need to be conducted before it was used in the related geospatial applications [31, $\underline{32}]$.

\subsection{ASTER GDEM}

The ASTER GDEM is an optical DEM dataset that was first released in 2009, generated based on measurements collected by the ASTER sensor onboard NASA's Terra platform. It was the first open-source global elevation dataset with a 1 -arc-second resolution and a global scale $\left(83^{\circ} \mathrm{S}-83^{\circ} \mathrm{N}\right)$. However, the quality of the first version was unsatisfactory. According to the results reported by a joint U.S./Japan validation team, the overall accuracy of the GDEM v1.0 data is around $20 \mathrm{~m}$ at the 95\% confidence level []. ]. The updated version was released in 2011, with resolution improvement, artefact reduction, and water-body editing, incorporating $~ 260,000$ supplementary scenes. The 
spatial resolution of the ASTER GDEM v2 is $\sim 30 \mathrm{~m}$ referenced to WGS84/EGM96, with an accuracy of $17 \mathrm{~m}$ at the $95 \%$ confidence level [33]. More recently, the generation team have been working on GDEM v3 with an additional 350000 ASTER scenes incorporated and better water-body delineation [르].

\subsection{ALOS AW3D DEM}

The ALOS was launched in 2006, and the mission ended in 2011. Equipped with the Panchromatic Remote-sensing Instrument for Stereo Mapping (PRISM), ALOS was able to record a high-resolution 3-D view of the Earth's surface $[4, \underline{35}]$. The current open-source release refers to "ALOS World 3D - 30m (AW3D30)", which is the resampling result generated from the ALOS 5-m DEM published since 2015 [ $\underline{36}$ ]. The AW3D30 v1.0 was released in 2016 within $82^{\circ} \mathrm{S}-82^{\circ} \mathrm{N}$ latitudes. With the large number of voids existing in the version 1 data, the updated AW3D30 v1.1 filled the void pixels within $60^{\circ} \mathrm{S}-60^{\circ} \mathrm{N}$ by merging with the existing DEMs [37]. The latest update were released recently, referring to AW3D30 v2.2, with 14535 tiles improved by the process of absolute offset errors from the ICESat reference and relative striping errors along the satellite orbits by updated calibration between $60^{\circ} \mathrm{S}$ to $60^{\circ} \mathrm{N}$, and northern region over $60^{\circ} \mathrm{N}$ improved [38].

\subsection{ICESat GLAS}

During 2003-2009, the Geoscience Laser Altimeter System (GLAS) carried onboard ICESat obtained along-track laser footprints recording the land topography all over the world [39]. The spatial diameter of the laser spots on the Earth's surface was approximately $70 \mathrm{~m}$, and the spacing between the along-track points was $\sim 170 \mathrm{~m}$, while the cross-track spacing varied from several kilometers to several tens of kilometers [ㅁ]. Over the flat regions, the vertical accuracy of the GLAS points (GLAS/ICESat L2 Global Land Surface Altimetry Data Version 34, GLAH14) can be better than $10 \mathrm{~cm}$ [11]. Thus, the high-accuracy ICESat GLAS data are widely used as a reference in the vertical evaluation of DEM data [ $\underline{42}, \underline{43}]$. However, there might still be outliers caused by poor acquisition conditions (e.g. cloud reflections and saturated waveforms), which need to be filtered [느].

\subsection{Other fused DEM datasets}

Despite the DEM products introduced above, there were some other merged DEM datasets reconstructed by blending the existing products worth to mention. The earliest global data product was the 1-km GTOPO30 DEM compiled from multi-source raster and vector topographic information [45]. With the development of remote sensing techniques, the USGS and the NGA developed the Global Multi-resolution Terrain Elevation Data 2010 (GMTED2010) product suite reconstructed from elevation data with higher resolution to replace GTOPO30 in global- and continental-scale applications [ㅌ]. GMTED is a multi-resolution data suite with elevation products for each of the 30-, 15-, and 7.5-arc-second resolutions. However, these coarse-resolution datasets are not adequate for the refined monitoring of the Earth.

The SRTM DEM is one of the earliest open-source quasi-global DEM datasets with a relatively stable quality. Thus, most of the fusion works have been based on SRTM data, and have focused on the quality problems, including data voids and anomalies (gross errors), as well as random and systematic errors. As a result, the majority of the fused datasets are of 3-arc-second resolution, which is consistent with the previously released SRTM3 data. Some representative works include the collection of Viewfinder Panorama (VFP) DEMs [ㅍ]], the Global Land Survey Digital Elevation Model (GLSDEM) [20], the EarthEnv-DEM90 [19] and the MERIT DEM [21]. The detailed information of the representative fused DEM datasets introduced in this section were given in Table 1. 
Table 1. Detail information of some representative fused DEM datasets given in Section 2.5.

\begin{tabular}{ccccc} 
& Time & Coverage & Resolution & Main data source \\
\hline GTOPO30 & 1996 & S90-N90 & $1 \mathrm{~km}$ & $\begin{array}{c}\text { Multiple raster and vector sources of topographic } \\
\text { information }\end{array}$ \\
\hline GMTED2010 & 2010 & S90-N90 & $\begin{array}{c}225 \mathrm{~m}, 450 \mathrm{~m}, \\
1 \mathrm{~km}\end{array}$ & $\begin{array}{c}\text { SRTM3, Canadian elevation data, SPOT5 } \\
\text { Reference3D, ICESat GLAS }\end{array}$ \\
\hline GLSDEM & 2013 & S60-N83 & $90 \mathrm{~m}$ & SRTM3, GTOPO30, NED, CDED \\
VFP-DEM & 2014 & S90-N90 & $90 \mathrm{~m}$ & SRTM3, ASTER GDEM \\
\hline EarthEnv-DEM90 & 2014 & S60-N83 & $90 \mathrm{~m}$ & SRTM3, ASTER GDEM, GLSDEM \\
\hline MERIT DEM & 2017 & S60-N82 & $90 \mathrm{~m}$ & SRTM3, AW3D30, VFP-DEM, ICESat GLAS \\
\hline
\end{tabular}

\section{The generation of GSDEM-30}

With the public release of some new datasets, such as the SRTM1 and AW3D30 DEM, there is a chance to reconstruct a high-quality DEM dataset combining the advantages of the multi-source DEMs with a higher resolution and better accuracy. In this section, we introduce the generation of GSDEM-30, which is mainly reconstructed from the 30-m unfilled SRTM1 DEM, the ASTER GDEM $\mathrm{v} 2$, and AW3D30 using the proposed fusion strategy. The ASTER GDEM and AW3D DEM derived from optical observations are inevitably influenced by the clouds/cloud shadows. Thus, we used the SRTM1 as the base, and the quality issues were handled by the fusion of auxiliary information. Considering the global data characteristics, data processing techniques varied with latitudinal zone. The flowchart of the GSDEM-30 generation is shown in Fig. 1.

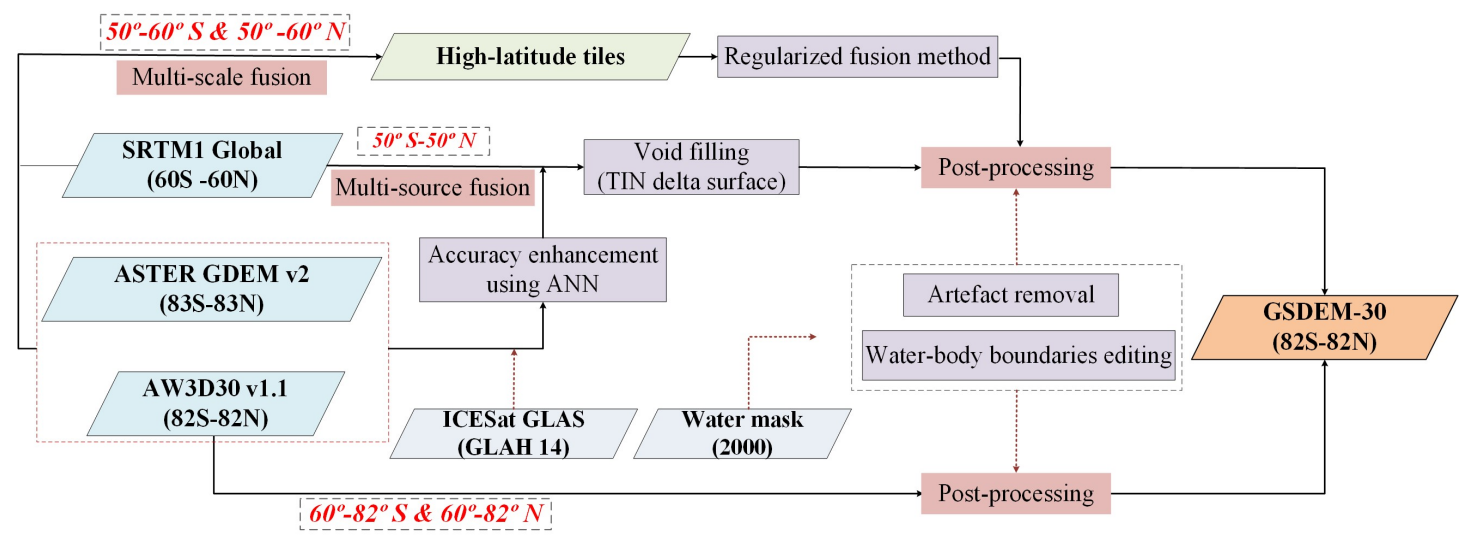

Fig. 1 Flowchart of the generation of GSDEM-30.

\subsection{Multi-source fusion for the void tiles}

Considering the relatively good quality of the SRTM1 data over the flat areas, we used the ASTER GDEM v2 and AW3D30 DEM to fill the voids within the SRTM1 tiles, and generate a seamless dataset. In most cases, the accuracy over the areas that are difficult to observe is relatively poor. Thus, artificial neural network (ANN)-based accuracy enhancement was firstly employed to capture the auxiliary elevation values within the voids [2].

ICESat GLAS points were used as the target reference in the accuracy enhancement. Firstly, the GLAS points were filtered and registered to WGS84/EGM96. The filtering parameters were similar to those used in previous works [녀] , where the 6-peak points were first removed to exclude the 
points with a saturated waveform. Moreover, the selected points should satisfy the criteria where the received energy was lower than $10 \mathrm{fJ}$ and the signal width was less than $25 \mathrm{~m}$. Furthermore, if the absolute height difference between the GLAS values and the corresponding elevation measurements of any DEM product among the datasets involved in the fusion was less than $100 \mathrm{~m}$, the GLAS points were retained.

The multi-source fusion method composed of accuracy enhancement and void filling process is displayed in Fig. 2. The elevation values, the slope index and the spatial location (latitude and longitude coordinates) jointly constructed the training dataset for the ANN model, while the corresponding GLAS point was the target. The statistical relationship between the input and the targeted elevation with high accuracy were learned from the training dataset with ANN, and the height values to be corrected were then processed adaptively according to the learned spatial pattern that varied with topography. Due to the sparse distribution of GLAS data compared with the gridded elevation data, this is a point-surface fusion process [뇨]. With the corrected surface obtained, the triangular irregular network (TIN) delta surface void filling method was used to eliminate the bias between the elevation data to be filled and the corrected terrain surface [49]. The delta surface is the difference between the actual terrain surface and the base surface, which is an interpolated surface using the values bordering the voids. For example, the delta surface $S_{\Delta A}=S_{A}-S_{b_{A}}$ is calculated with the actual elevation $\left(S_{A}\right)$ and the interpolated base elevation $\left(S_{b_{A}}\right)$ of the auxiliary data for the void area. This method is mainly based on the assumption that the local surface trend is similar for multi-source observations over the same topographic region. As a result, the void filled data surface $S_{f}$ can be approximated to $S_{f}=S_{b}+S_{\Delta A}$, where $S_{b}$ is the base surface of the data with voids (SRTM1 data).

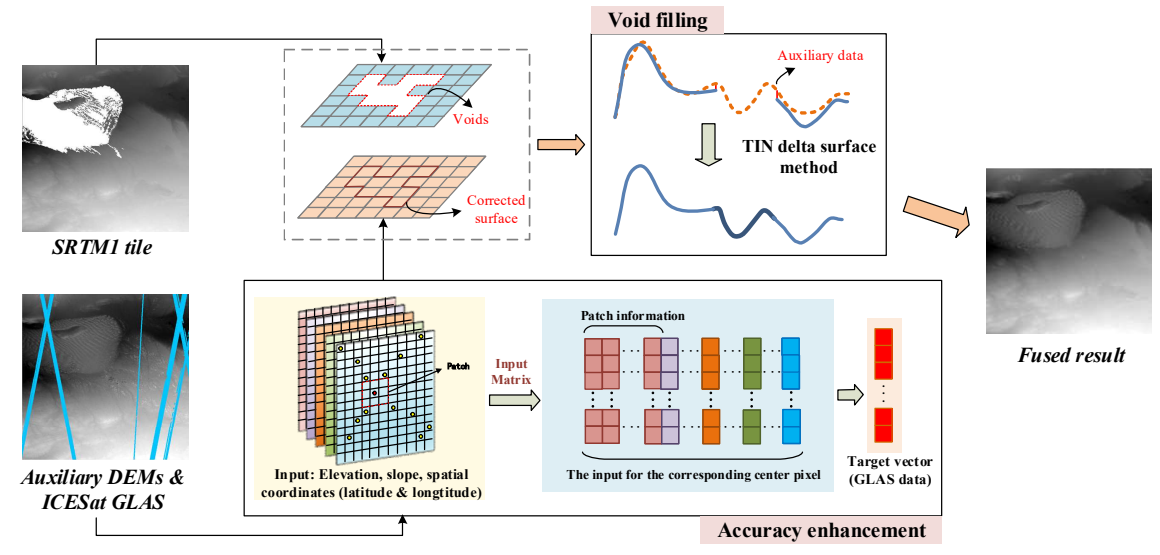

Fig. 2 The workflow of the multi-source fusion method for the void tiles.

In the generation process, the SRTM1 data tiles with voids between $50^{\circ} \mathrm{N}$ and $50^{\circ} \mathrm{S}$ were handled tile-by-tile $\left(1^{\circ} \times 1^{\circ}\right)$ following the accuracy enhancement and void filling process. The auxiliary data for void filling were selected from the ASTER GDEM v2 and AW3D30, depending on the local data quality. Specifically, if the vertical accuracy or visual performance of AW3D30 data was evidently abnormal, the ASTER GDEM tile was used as the replacement.

\subsection{Multi-scale fusion for the high-latitude tiles}

For SRTM1 data beyond the $50^{\circ}$ latitude, the tiles are sampled at 2 arc-seconds along the longitudinal direction. To ensure the spatial consistency of the global data, we used the multi-scale data fusion method [26] to up-sample the high-latitude data between $50^{\circ}$ and $60^{\circ}$ to 1 -arc $\times 1$-arc tiles. To reconstruct the high-latitude SRTM tiles, the input data include the original SRTM1 and the high-resolution ASTER GDEM and AW3D30 data.

The multi-scale fusion method employed is a regularized method based on the assumed generative model $\boldsymbol{y}_{k}=\boldsymbol{A}_{k} \boldsymbol{u}+\boldsymbol{n}_{k}$, where $\boldsymbol{u}$ is the desired high-resolution DEM, and $\boldsymbol{y}_{k}$ is the 
input multi-scale data generated from $\boldsymbol{u}$ after degradation. The degradation matrix $\boldsymbol{A}_{k}$ is used to simulate the relationship between the input and the reconstructed data. Generally, the degradation describes the pixel misalignment between the input $\boldsymbol{y}_{k}$ and the referenced high-resolution grid, the down-sampling effect and different spatial coverage of the input data. Moreover, there might be invalid voids and anomalies in the DEM data, which should be excluded from the reconstruction. As a result, $\boldsymbol{A}_{k}$ is the multiplication of the translation matrix $\boldsymbol{M}_{k}$, the sampling matrix $\boldsymbol{D}_{k}$ and the cropping matrix $\boldsymbol{O}_{k}$, which can be described as $\boldsymbol{A}_{k}=\boldsymbol{O}_{k} \boldsymbol{D}_{k} \boldsymbol{M}_{k}$ [26]. Besides, $\boldsymbol{n}_{k}$ stands for the additive noises in the input DEMs, also including the model errors. Based on the generative model, the problem of reconstructing $\boldsymbol{u}$ is an inverse problem, given the input multi-scale DEMs known and the desired reconstructed DEM unknown. Thus, the regularized function can be built to get the fusion result:

$$
\hat{\boldsymbol{u}}=\arg \min \left[\sum_{k} w_{k} \cdot\left\|\left(\boldsymbol{y}_{k}-\boldsymbol{A}_{k} \boldsymbol{u}\right)\right\|_{1}^{1}+\lambda \sum_{i} \sum_{t=1}^{\tau}\left\|d_{c}^{t}\left(u_{i}\right)\right\|_{p_{i}}^{p_{i}}\right]
$$

In Equation (1), the first term is the fidelity providing the constraint for the conformance of the reconstructed data to the input observations. With multiple input data with different scales, the $l_{1}-$ norm is used as the constraint function for the fidelity, thus minimize the influence of horizontal misalignments between the inputs. Moreover, a weight is assigned to each input DEM data, thus the data with higher quality make a greater contribution in the fusion process. The weight $w_{k}$ is calculated based on the fidelity residual during the numerical iteration of minimizing the objective function (1), which can be expressed as:

$$
w_{k}^{(r+1)}=K \cdot \frac{1 / \log \left(1+\left\|\boldsymbol{y}_{k}-\boldsymbol{A}_{k} \boldsymbol{u}^{(r)}\right\|_{2}\right)}{\sum_{k=1}^{K}\left[1 / \log \left(1+\left\|\boldsymbol{y}_{k}-\boldsymbol{A}_{k} \boldsymbol{u}^{(r)}\right\|_{2}\right)\right]}
$$

This function determines the contribution of each DEM data among the $K$ input observations in the fusion, where $w_{k}^{(r+1)}$ is the updated weight for the current $r$ th iteration calculated using the results obtained in the last iteration.

The second term is the regularization prior used to describe the spatial characteristics of the fused data. Here we employ the adaptive Markov-random field regularization to model the contextual correlations between neighboring elevation pixels [26]. For $d_{c}(\cdot)$ in equation (1), the finite-difference approximations to second-order derivatives in four directions $(\tau=4)$ are employed to measure the spatial relationship between the neighboring pixels [미]. The key point is to adapt the prior model to data spatial characteristics considering the terrain undulation. Thus, the adaptive constraint function is designed for the derivatives based on local slope information. The smooth $l_{2}-$ norm is used to suppress the data noise and artefacts for the flat terrain, while the $l_{1}-$ norm is employed for the regions with relatively sharp terrain features. Thus, the constraint norm for the pixel $i$ is defined as:

$$
p_{i}= \begin{cases}2 & S_{i}<T_{s} \\ 1 & S_{i} \geq T_{s}\end{cases}
$$

where $S_{i}$ is the normalized slope value for the pixel $i$, and $T_{s}$ is the empirical threshold to distinguish different terrain features. The tradeoff between the two competing terms in (1) can be tuned by the regularization parameter $\lambda$. To get the fusion result, the objective function can be efficiently solved by the iteratively reweighted norm algorithm and the preconditioned conjugate gradient (PCG) method, as illustrated in [ㄷ]. This fusion method can enhance the terrain details effectively with high-resolution auxiliary information involved and different data quality considered. 
4 of 21

\subsection{Post-processing}

By integrating the multiple observations, most of the voids could be filled with effective elevation values using the fusion method described in Sections 3.1 and 3.2. However, some anomalies were still difficult to eliminate in large-scale cloud-covered and shaded observations. Thus, post-processing was necessary. The filled data tiles were examined iteratively, and the detected anomaly pixels were filled using statistical interpolation methods. For the initial version, simple methods such as thin plate splines and triangular interpolation were employed. Moreover, The 30-m global inland water mask extracted using Landsat images was employed to edit the DEMs with low quality around water bodies [51]. Due to the coverage limit of SRTM data, the high-latitude no-data region (above $60^{\circ} \mathrm{N}$ ) was filled with the AW3D30 data. The final GSDEM-30 product is a void-filled and accuracy-improved global seamless DEM dataset with a 1-arc-second resolution, which we refer to as GSDEM-30. The GSDEM-30 data $\left(82^{\circ} \mathrm{S}-82^{\circ} \mathrm{N}\right)$ are currently available from http://sendimage.whu.edu.cn/res/DEM_share/.

\section{Accuracy validation and analysis}

\subsection{Auxiliary data for evaluation}

In this paper, the quality of the global DEM products is assessed comprehensively. Except for the vertical accuracy evaluation over the global land surface with GLAS data, the U.S. NED DEM was used as the reference for the local validation [52]. The NED DEM is a bare-earth elevation product produced by the USGS, which provides high-quality elevation information covering the continental United States, Alaska, Hawaii, and the island territories. This is a multi-resolution DEM dataset referenced to the North American Datum of 1983 (NAD 83)/North American Vertical Datum of 1988 (NAVD 88), where the national-scale resolution is 1 arc-seconds. Higher-resolution data with 1/3-arc-second ( $\sim 10 \mathrm{~m})$ and 1/9-arc-second $(\sim 3 \mathrm{~m})$ spacing are also available for some tiles.

For the landscape analysis of the error distribution, the GlobeLand 30 data produced by the Chinese global land-cover mapping project were introduced for the land-cover extraction [53]. This is a dataset with 10 classes for the land cover of the Earth between $80^{\circ} \mathrm{S}$ and $80^{\circ} \mathrm{N}$. The overall accuracy of GlobeLand30-2010 has been reported to reach $80.33 \%$, validated with over 150000 test samples [푸].

Considering the previous accuracy reports [36, $\underline{55}]$, the 30-m ASTER GDEM v2 and AW3D30 DEM were included in the evaluation for comparison with the GSDEM-30 dataset. Due to the limited spatial coverage of the source SRTM data, the high-latitude areas (above $60^{\circ} \mathrm{N}$ and below $60^{\circ} \mathrm{S}$ ) of GSDEM-30 were filled directly with the AW3D30 DEM. Therefore, the accuracy analysis in Sections 4.2 and 4.3 mainly concentrate on the common quasi-global land areas between $60^{\circ} \mathrm{S}$ and $60^{\circ} \mathrm{N}$. The analysis about the high-latitude data are presented in Section 4.4.

\subsection{Global error distribution}

\subsubsection{Accuracy assessment}

The accuracy of the DEM datasets is mainly assessed using the global ICESat GLAS data points as the reference. Using the same validation data, it is possible to compare the accuracy performance of the different DEM products. The selection criteria of GLAS data was introduced in Section 3.2.1. Over 227M GLAS points participated in the accuracy validation. The performance of the multi-source accuracy enhancement method has been validated in [22], where the independent validation results was comparative with the training performance. Furthermore, the GLAS data were only used to correct the void pixels in our work. Therefore, the global accuracy validation with GLAS points are feasible and will not lead to a biased result.

Firstly, the error histograms of all the continental measurements are given in Fig. 3, to show the distribution of the difference between the GLAS points and the DEM products (ICE-DEM). The ideal error bar is approximated to a normal distribution with a small standard deviation. It can be clearly 
observed that the error distribution of the ASTER GDEM is the most disparate, and the ratio of points with an absolute error smaller than $2 \mathrm{~m}$ is only $32.7 \%$. Compared with the ASTER GDEM data, which are seriously affected by noise, the other two datasets show a much better accuracy. From the analysis of the results, the 90th percentile absolute error of the AW3D30 is slightly smaller than that of GSDEM-30, while the ratio of points with a small absolute error $(<2 \mathrm{~m})$ shows the opposite trend. The error distributions of these two datasets are very similar. Besides, the errors of the AW3D DEM and GSDEM-30 are mainly negative, and are likely caused by the systematic bias, and the overestimation of the bare-earth height over the vegetated and rugged areas. This is consistent with the local study in [ㄷ6], where the distribution of ICESat centroid height minus SRTM elevations show a peak around zero, and become more evidently negative with the increase of tree cover and terrain roughness.

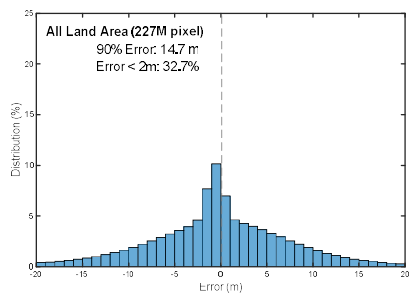

(a) ASTER GDEM

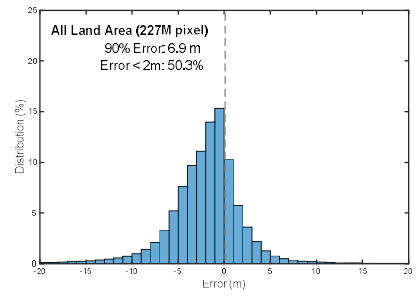

(b) AW3D30 DEM

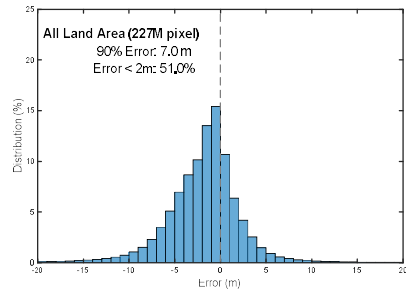

(c) GSDEM-30

Fig. 3 The error histogram of the points over all the land areas between $60^{\circ} \mathrm{S}$ and $60^{\circ} \mathrm{N}$ (dif: ICE-DEM).

In addition to the error statistics, we also present the $0.25^{\circ} \times 0.25^{\circ}$ global distribution of the error difference, with regard to the root-mean-square error (RMSE) and MEAN (mean error) values, respectively (Figs. 4-5) [22]. The error statistics were averaged with the points within the corresponding grid cell. From the results, we did analyze the global variance of the DEM data quality. All the error distribution maps show that the main accuracy problems are found in the southern Tibet Plateau, the western coastline of South America, and the island areas around the globe. Thus, the comparison of the different products focuses on the high-altitude plateaus, coastline areas, and high-latitude areas around the globe. The global mapping results also reveal that the ASTER GDEM performs the worst in data accuracy, with relatively large errors across the land areas, especially over the vegetated and desert areas. This is basically consistent with the previous studies, in that the quality of the ASTER GDEM is seriously influenced by random noise and artefacts [24]. Despite the poor performance of the ASTER GDEM, the error maps of the other two datasets are in good agreement with each other. The error maps of the AW3D30 and GSDEM-30 data show a very similar spatial distribution. The main difference can be found where larger biases exist in the AW3D30 DEM in North America, Africa and the high-latitude areas above $50^{\circ}$.

In terms of the map of MEAN values (Fig. 5), we can get more information about the positive or negative bias of the DEM products. The spatial distribution of the MEAN values is generally consistent with the absolute difference map (Fig. 4), which has a high correlation with the relief topography and vegetation cover. It can be observed that all the DEM elevations have a significant trend of overestimation in the high-mountain regions. This could be caused by the large sub-pixel variability over the rugged topography, considering the different scales for the DEM grid and the GLAS points. 
6 of 21

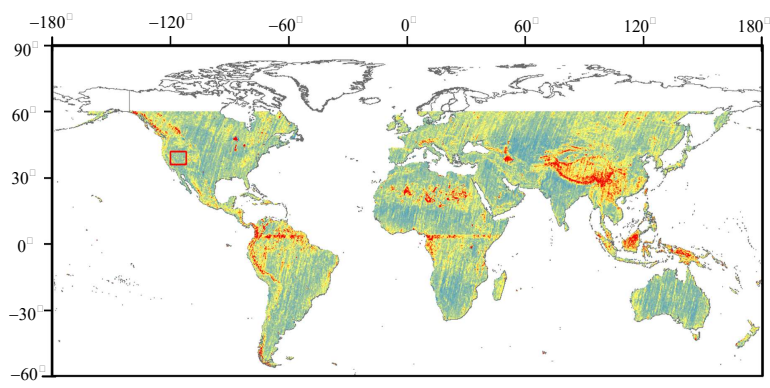

(a) ASTER GDEM

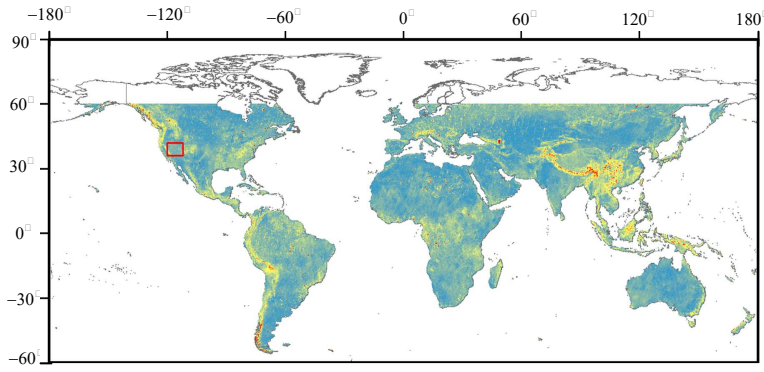

(b) AW3D30 DEM

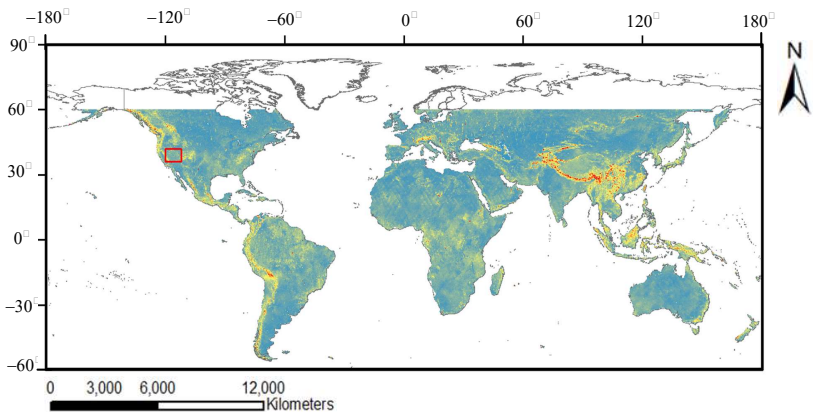

(c) GSDEM-30

Error RMSE
Unit : $m$

Fig. 4 The absolute difference between the ICESat GLAS data and the DEM products over the quasi-globe $\left(60^{\circ} \mathrm{S}-60^{\circ} \mathrm{N}\right)$. (a)-(d) The RMSE values for the $0.25^{\circ} \times 0.25^{\circ}$ tiles of the DEM datasets. The area in the red rectangle was zoomed in Fig. 7 for a detailed analysis.

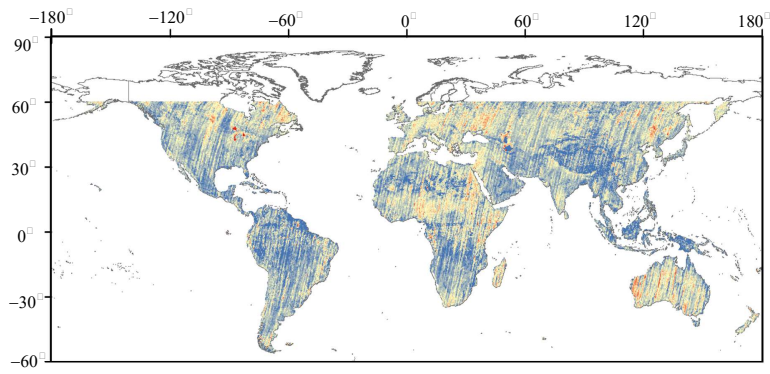

(a) ASTER GDEM 


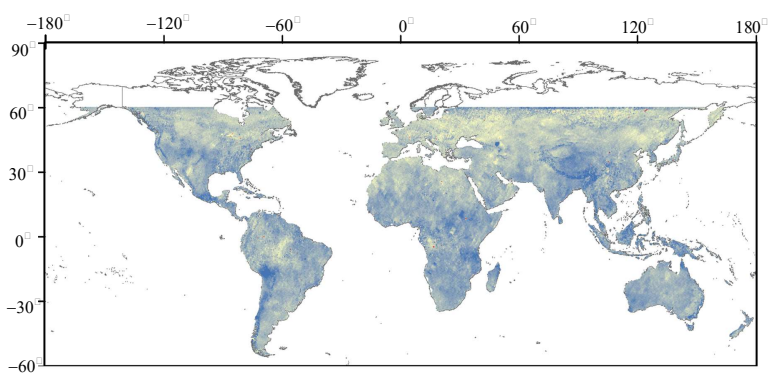

(b) AW3D30 DEM

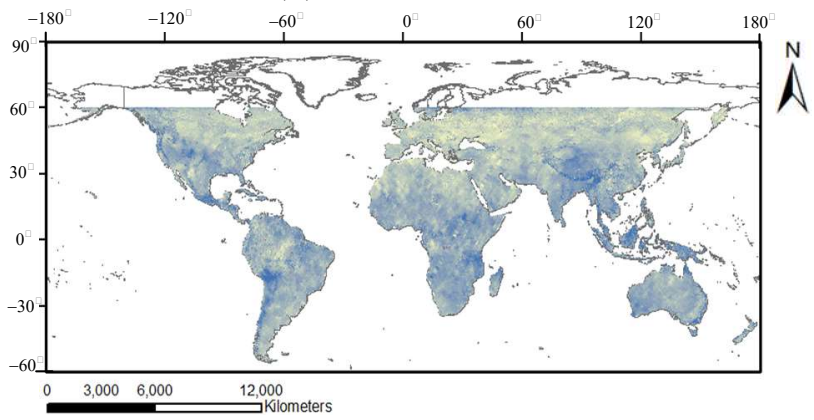

(c) GSDEM-30

Error MEAN
Unit $: m$

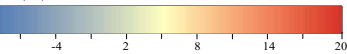

Fig. 5 The difference between the ICESat GLAS data and the DEM products over the quasi-globe $\left(60^{\circ} \mathrm{S}-60^{\circ} \mathrm{N}\right) .(\mathrm{a})-(\mathrm{c})$ The MEAN values for the $0.25^{\circ} \times 0.25^{\circ}$ tiles of the DEM datasets.

To validate the accuracy assessment results based on ICESat GLAS data, we chose the 10-m high-quality NED data for the local validation. We chose an area with significant differences between the error maps in North America for the sample validation, where the extent was indicated by the red rectangle area in Fig. 4 . The NED data within the sample area and the difference maps of the DEM datasets were presented in Fig. 6. Before calculating the difference maps, we converted the reference datum of the DEM products from WGS84/EGM96 to NAD 83/NAVD 88, to be consistent with the NED data. The NED data were then resampled to $30 \mathrm{~m}$ using the bilinear interpolation algorithm, and sub-pixel registration was conducted to ensure that the validated datasets were aligned with the NED data. The difference maps showed that GSDEM-30 had the closest agreement with the NED data. Comparatively, the ASTER GDEM and AW3D30 data were obviously affected by stripe errors. The local validation results were consistent with the global validation with ICESat GLAS points (shown in Fig. 4 and Fig. 6 (e)), which made the global comparison credible. 

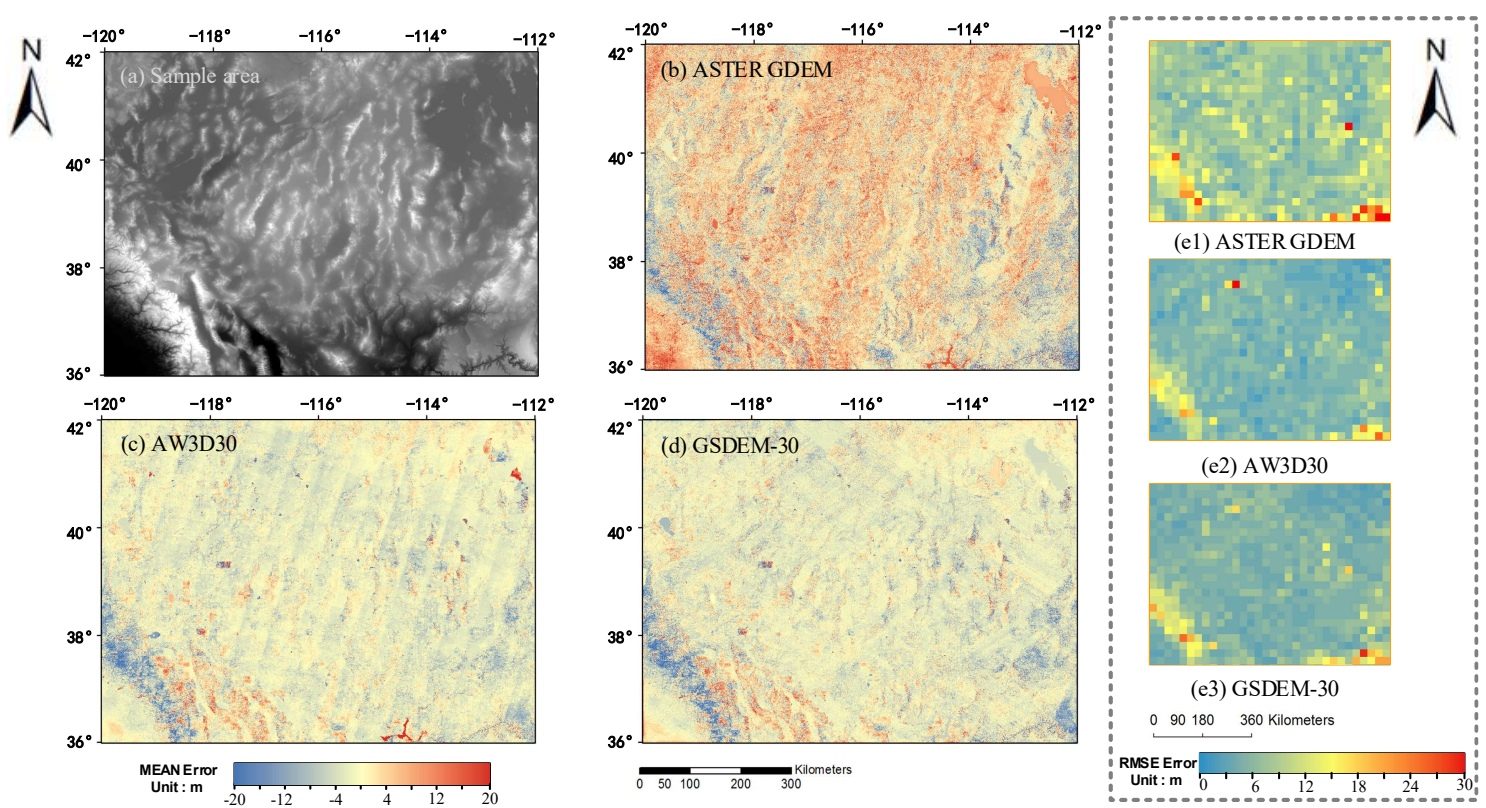

Fig. 6 The difference map between the NED data and the different DEM datasets. (a) The NED data within the sample area (elev: -85 to $4414 \mathrm{~m}$ ). (b)-(d) indicate the difference maps between the NED data and the ASTER GDEM, AW3D30 DEM, and GSDEM-30, respectively. (e) Zoomed view of the highlighted region of the global RMSE distribution shown in Fig. 5.

\subsubsection{Error distribution for landscapes}

For a more comprehensive analysis of the DEM quality, we further examined the distribution of elevation errors corresponding to different landscapes. The GlobeLand30 data were introduced for the global land-cover classification, with 10 land-cover types classified all over the world (Table 2). We extracted the corresponding land-cover types of all the global validation points, and then calculated the statistical information for each class, as shown in Figs. 7-8. In Fig. 7, the gray bars show the 90th percentile of the absolute error, and the blue curves indicate the number of points for the land-cover type. Fig. 8 presents the ratio of points with absolute errors of less than $2 \mathrm{~m}$, and the RMSE values for the different landscapes.

Table 2 The land-cover types of the GlobeLand30 data.

\begin{tabular}{cccccc}
\hline \hline Class order & 1 & 2 & 3 & 4 & 5 \\
\hline Class & Cultivated land & Forest & Grassland & Shrubland & Water bodies \\
\hline Class order & 6 & 7 & 8 & 9 & 10 \\
\hline Class & Wetland & Tundra & Artificial surfaces & Barren land & Permanent snow and ice \\
\hline \hline
\end{tabular}
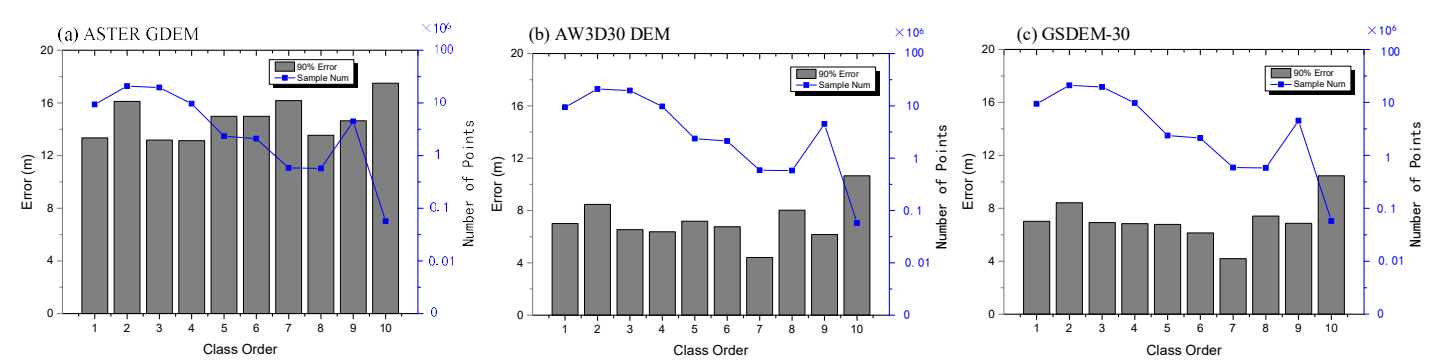

Fig. 7 The error histograms of the points corresponding to different land-cover types based on GlobeLand30. 
9 of 21
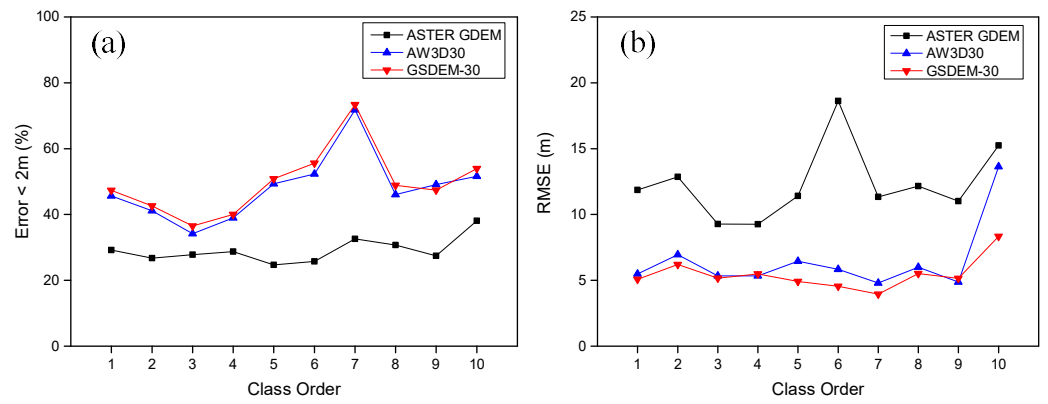

Fig. 8 The error curves of the different DEM products. (a) The ratio of points with an absolute error of less than $2 \mathrm{~m}$ within the class. (b) The RMSEs for the different land-cover types.

The data have a better quality over the flat areas, such as the grassland, tundra, and barren land. For the vegetated areas and artificial surfaces, the barren land can be covered with dense vegetation or buildings, where the errors tend to be larger. The errors for permanent snow and ice covered areas are higher than for the other classes. This might because glaciers are mainly distributed in the high-mountain and high-latitude areas, where the quality of the DEMs is relatively low. Overall, the comparisons are basically consistent with the previous results. Among the three DEM products, the ASTER GDEM shows significant errors for all the classes. The statistical accuracies of the AW3D30 DEM and GSDEM-30 are in close agreement with each other. For most of the classes, the ratios of the low-error points of the GSDEM-30 are slightly higher than for AW3D30, and the RMSE values of GSDEM-30 indicate a better performance (Fig. 8). As the RMSE can better reflect the influence of large errors than the mean absolute error (MAE), the difference between the indices indicate that the AW3D30 data are contaminated with more large-error elevation values without the processing of anomalies. This conclusion can also be reflected in the global error mapping results (Figs. 4-5). We further divided the points into two categories, which are the points over the flat areas (with a slope of less than $6^{\circ}$ ) and the points covered by vegetation (forest and shrubland). The results in Fig. 9 show that the flat pixels have higher accuracies, while the vegetated pixels are contaminated with higher errors [57]. The overall accuracy of AW3D30 and GSDEM-30 are comparative, while GSDEM-30 outperforms AW3D30 in terms of the ratio of small-error points.

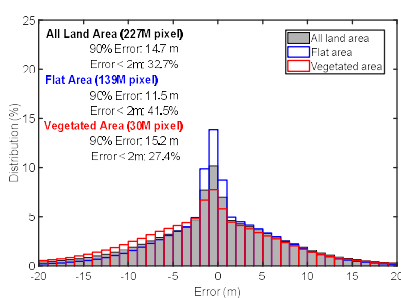

(a) ASTER GDEM

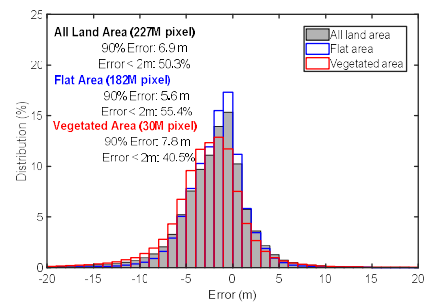

(b) AW3D30 DEM

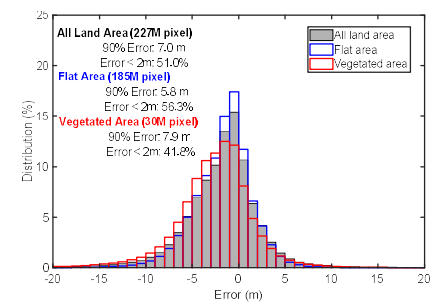

(c) GSDEM-30

Fig. 9 The error histograms of the points for all the land areas, flat areas, and vegetated areas (forest and shrubland).

\subsubsection{Height error propagation}

The global error assessment results given in Section 4.2.1 and Section 4.2.2 give us an overview of the DEM accuracy. However, this can only partly reflect the practical data quality, as it depends on the distribution of the ICESat GLAS points. Therefore, we employed an artefact detection technique, which we refer to as the MSA method, to delineate the influence of height errors propagated on the slope [27]. Due to the independence of the reference data, it is convenient to use this method to compare the artefact distribution of different DEM products.

Slopes are calculated over the cells within a given sub-region $\boldsymbol{\theta}\left(0.1^{\circ} \times 0.1^{\circ}\right.$ defined in this paper), and the maximum slope values are defined as $\max \left(\operatorname{slope}_{i, j}\right) \quad i, j \in \boldsymbol{\theta}$. If the maximum slope exceeds a given threshold, large artefacts may exist within the tile. Hirt [27] used the MSA 
method to examine the artefacts existing in the SRTM v4.1 and MERIT DEMs. In this paper, the MSA method is used to generate the global $0.1^{\circ} \times 0.1^{\circ}$ maximum slope map of the ASTER GDEM, AW3D30 DEM, and GSDEM-30 between $60^{\circ} \mathrm{S}$ and $60^{\circ} \mathrm{N}$. The results are shown in Fig. 10.

The maximum slope maps are partly consistent with the vertical error distribution maps (Figs. 4-5). The ASTER GDEM data are seriously contaminated with artefacts, and the error distributions of the AW3D30 DEM and GSDEM-30 show overall homologous trends. For the high-latitude regions (above $50^{\circ} \mathrm{N}$ ) and most of the flat areas (i.e. North America, South America, and eastern China), the GSDEM-30 data show a better performance. It can also be observed that some significant positive values, which indicate possible anomalies, can be found in the desert areas over central Africa in the AW3D30 DEM, which is consistent with the validation results obtained using ICESat GLAS points. The MSA method is mainly based on the assumption that the spurious artefacts can cause significant elevation changes between neighboring DEM cells. It has a limited ability to distinguish the artefacts and the natural terrain features in certain cases. Therefore, we conducted the manual examination of the detected tiles with a maximum slope exceeding the threshold $10 \mathrm{~m} / \mathrm{m}$, and showed the results in Fig. 11. According to the visual examination results, there are much more ASTER GDEM and AW3D30 data with anomalies contaminated than the GSDEM-30 data.

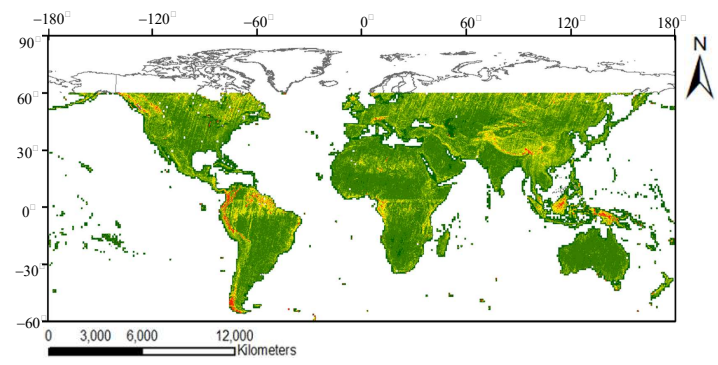

(a) ASTER GDEM

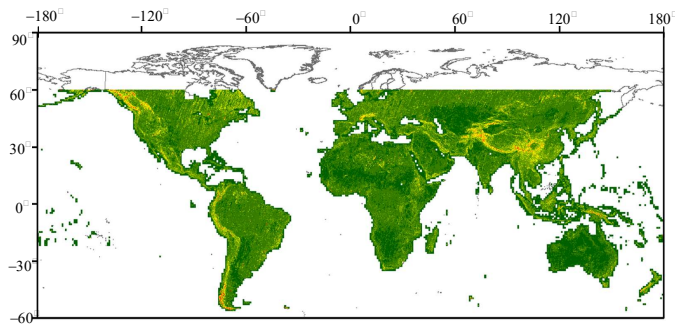

(c) AW3D30 DEM

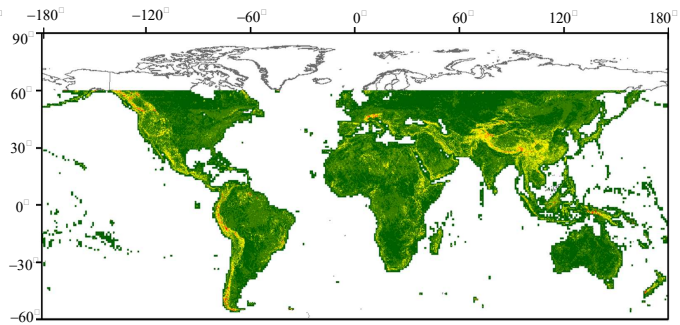

(d) GSDEM-30 Max Slope
Unit : degree

Fig. 10 The maximum slope distribution of the different DEM products over the quasi-globe $\left(60^{\circ} \mathrm{S}-60^{\circ} \mathrm{N}\right)$. The results are shown per $0.1^{\circ} \times 0.1^{\circ}$ tile.

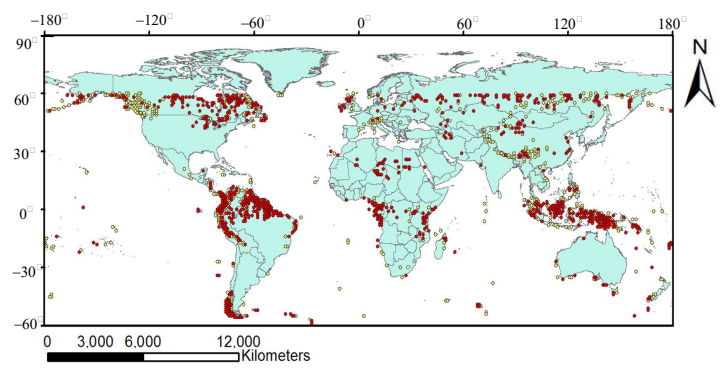

(a) ASTER GDEM 


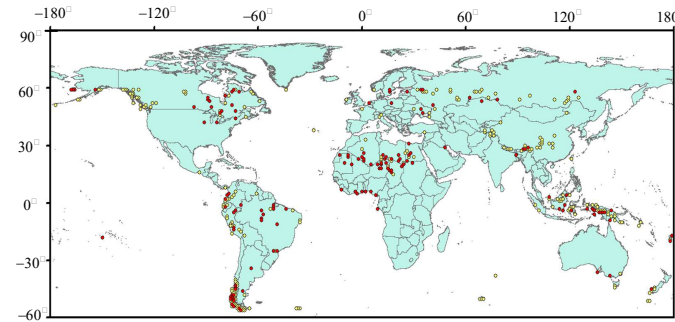

(b) AW3D30

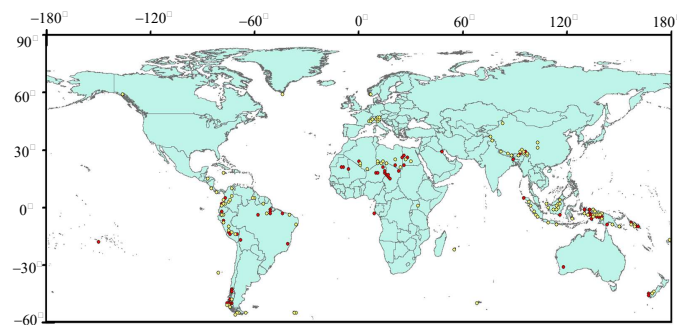

(c) GSDEM-30

Tiles with serious artefacts

Tiles with slight artefacts

Fig. 11. The artefacts distribution of global DEM datasets by visual examination $\left(60^{\circ} \mathrm{S}-60^{\circ} \mathrm{N}\right)$.

\subsection{Sample data validation}

In this section, local-scale assessment results are used to validate the global error distribution results presented in Section 4.2. The visual comparisons are the main focus to evaluate the delineation of terrain features, while the artefacts and multiple error components are examined throughout the multiple datasets over various topographies and land-cover types. Five classes are considered in this section, including woodland with dense vegetation covered regions, deserts, high-altitude rugged mountains, coastlands, and basins. For each class, 20 sample tiles were randomly selected from the ASTER GDEM, the AW3D30 DEM, and GSDEM-30, respectively. The sample tiles were selected world-wide within $60^{\circ} \mathrm{S}-60^{\circ} \mathrm{N}$, with the locations given in Fig. 12.

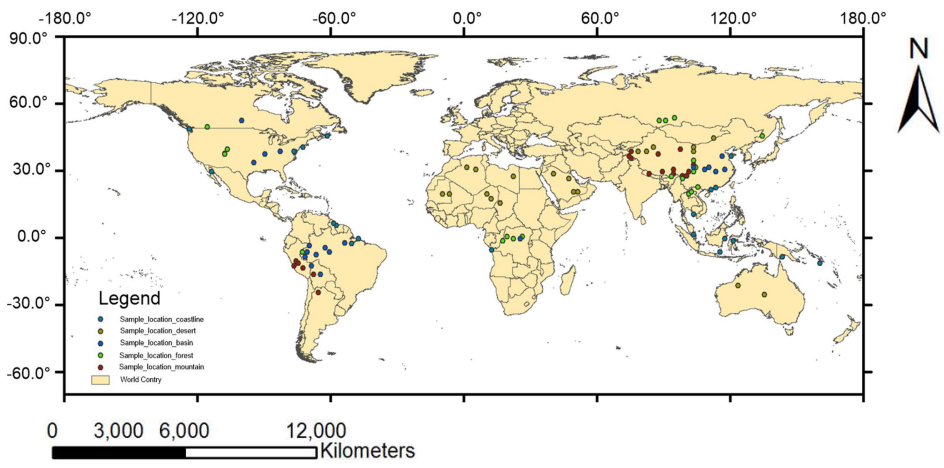

Fig. 12. The locations of the sample tiles.

In Fig. 13, we present the performance with regard to MAE, RMSE, and MEAN error calculated with the DEM data for the different landscapes referenced to the GLAS points. The ASTER GDEM shows the worst accuracy in terms of all the indices, while the vertical accuracy of the AW3D30 and GSDEM-30 data varies depending on the landscapes. For the desert areas, the AW3D30 DEM outperforms GSDEM-30, while GSDEM-30 performs better in mountainous regions. The accuracy validation results show little difference in the mean values for the vegetated areas, coastlines, and basins between these two elevation products.

The MAE results are basically consistent with the RMSE results. However, the difference in the RMSE might be more significant, due to the larger weight assigned to the elevation anomalies in the calculation. Specifically, the mean MAE of AW3D30 is slightly smaller than GSDEM-30 in the mountainous areas, while the RMSE shows the opposite trend. The reason for this is that the elevation values in the rugged topography are easily contaminated with anomalies. GSDEM-30 is better at processing the elevation anomalies, which thus improves the accuracy. 


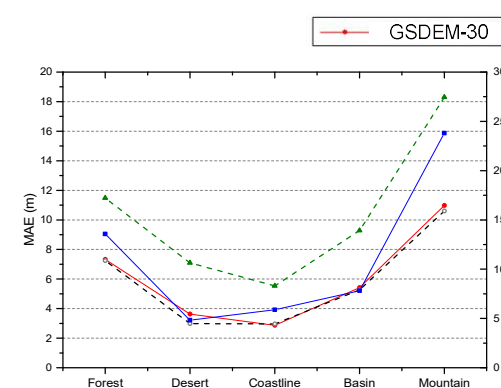

(a)

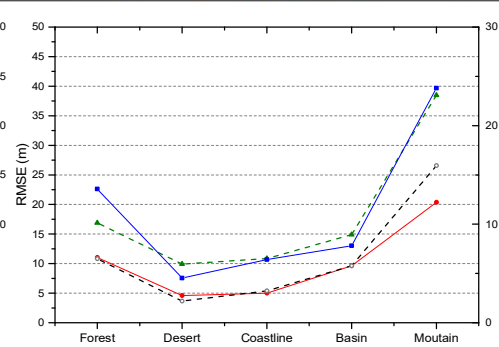

(b)

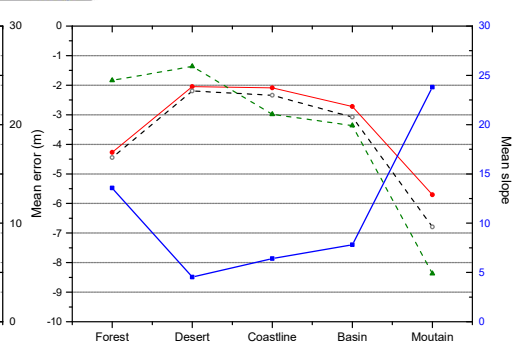

(c)

Fig. 13. The average error statistics for the sample tiles with different landscapes. (a)-(c) The error curves of the MAE, RMSE, and MEAN values, respectively.

A visual comparison is also useful to examine the data quality. In Figs. 14-18, we give some representative sample cases for the five classes of landscape feature. The results show that the ASTER GDEM data contain large amounts of artefacts, which is consistent with the accuracy validation results. In the majority of cases, the GSDEM-30 data perform the best in delineating the continuity of the terrain features, due to the good suppression of the elevation anomalies. That is because we examined the global data tiles carefully, and reproduced the tiles with large-scale anomalies, using the available auxiliary data in the generation of GSDEM-30. However, both the GSDEM-30 and AW3D30 data are contaminated by obvious stripe noise and anomalies in some flat areas (e.g., deserts and coastline areas).
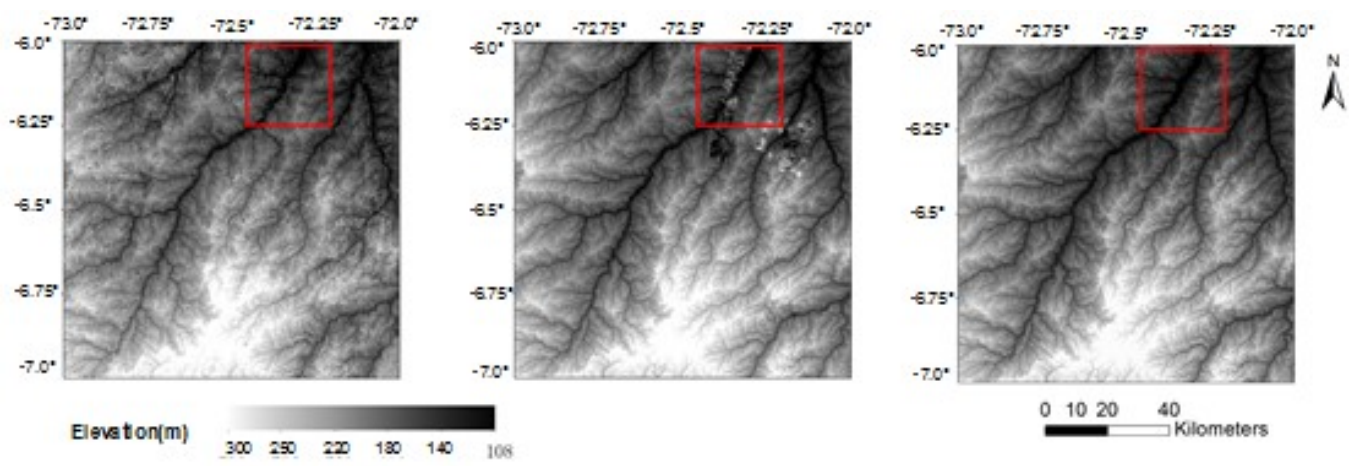

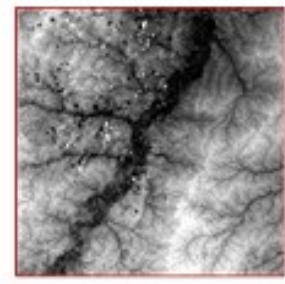

(a) ASTER GDEM

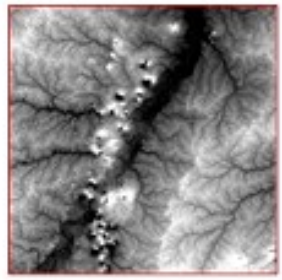

(b) AW3D30

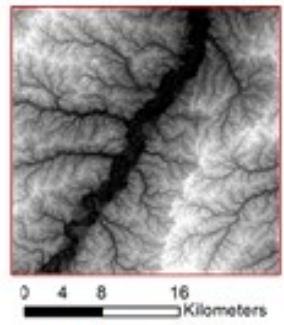

(c) GSDEM-30

Fig. 14. Visual comparison of the sample data in a forested area $\left(6-7^{\circ} \mathrm{S}, 72-73^{\circ} \mathrm{W}\right)$. 

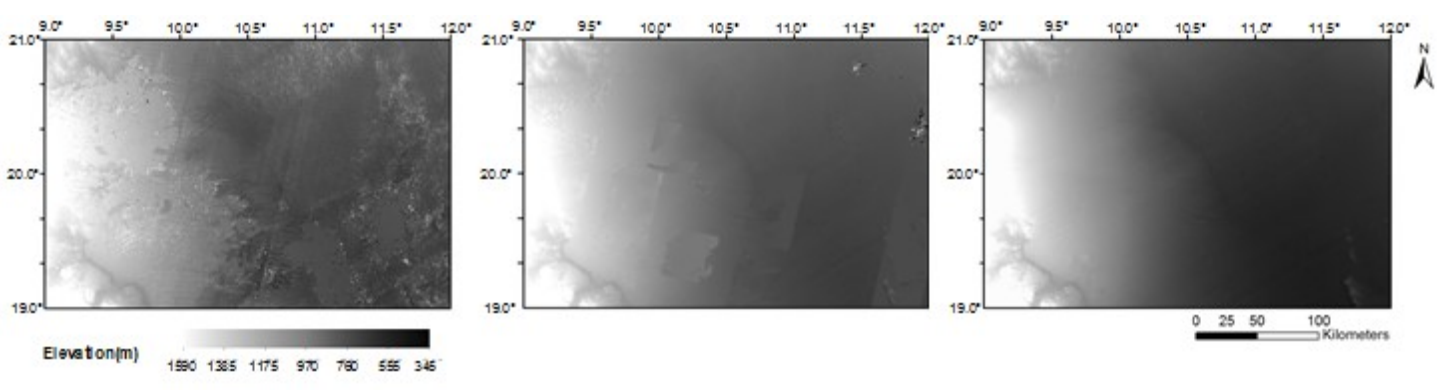

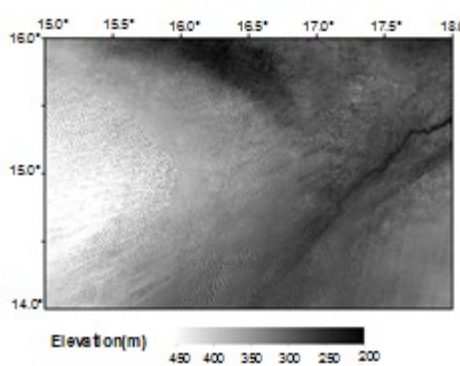

(a) ASTER GDEM

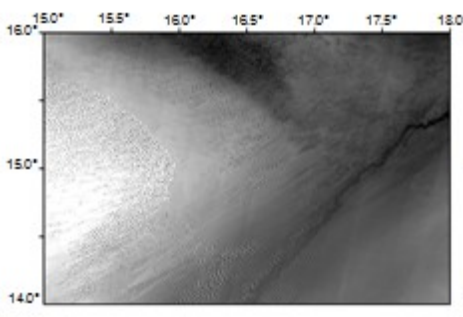

(b) AW3D30

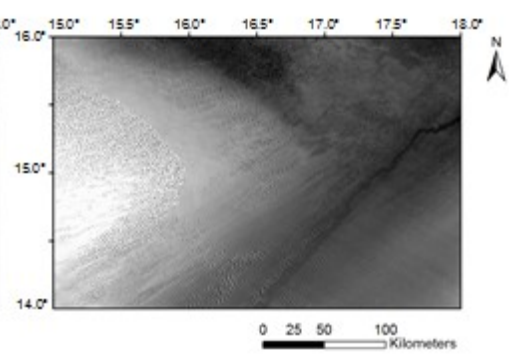

(c) GSDEM-30

Fig. 15. Visual comparison of the sample data in two desert areas (Sample area 1: $19-21^{\circ} \mathrm{N}, 9-12^{\circ} \mathrm{E}$, sample area $\left.2: 14-16^{\circ} \mathrm{N}, 15-18^{\circ} \mathrm{E}\right)$.

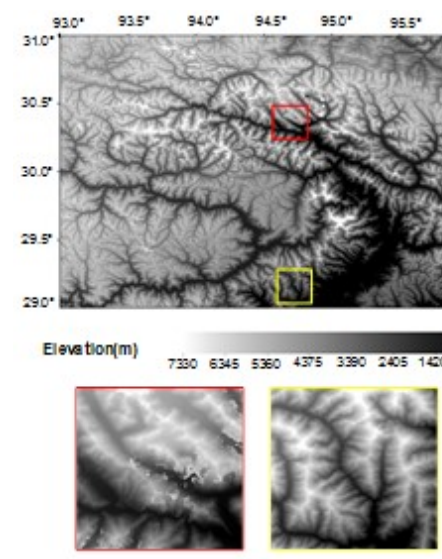

(a) ASTER GDEM

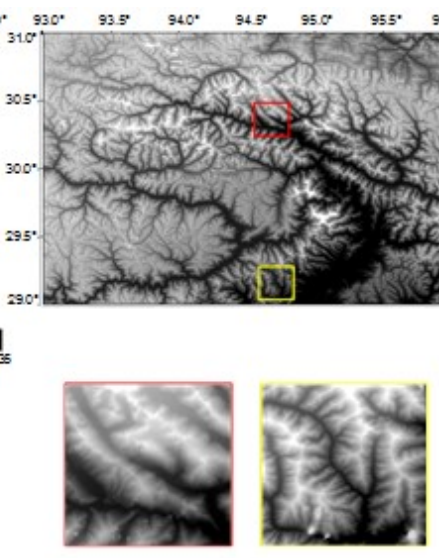

(b) AW3D30
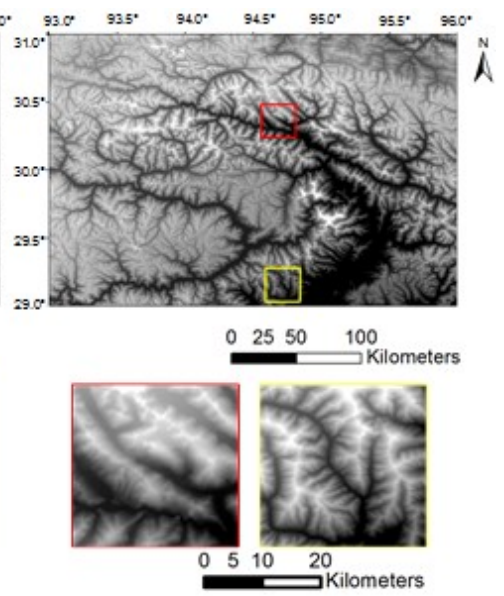

(c) GSDEM-30

Fig. 16. Visual comparison of the sample data in a mountainous area $\left(29-31^{\circ} \mathrm{N}, 93-96^{\circ} \mathrm{E}\right)$.

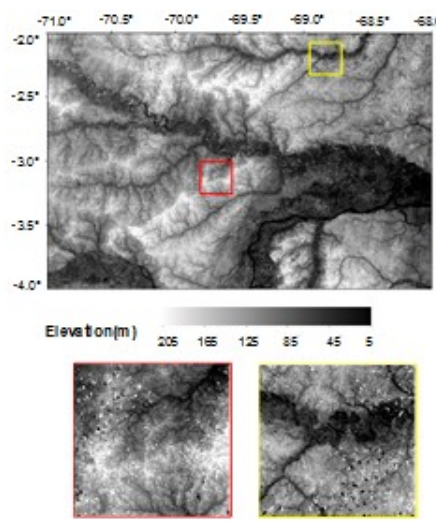

(a) ASTER GDEM
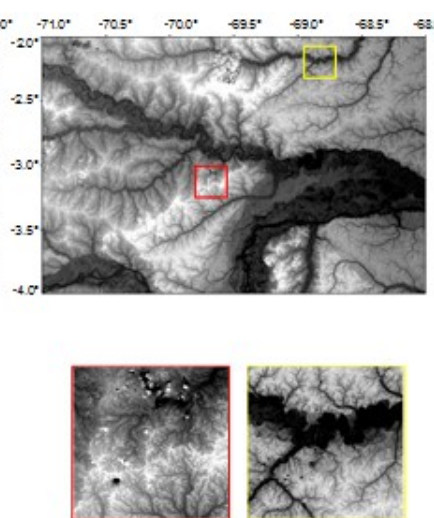

(b) AW3D30
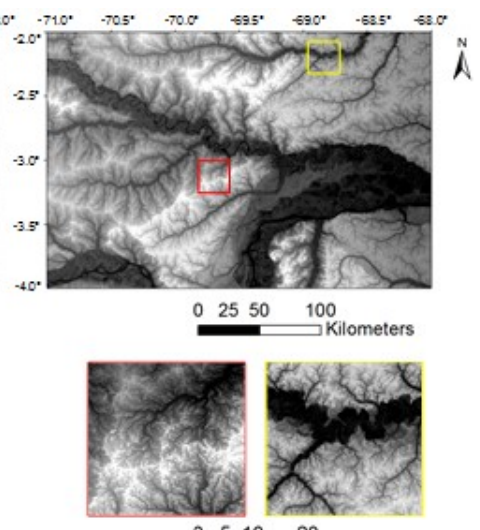

$0 \quad 510 \quad 20$

(c) GSDEM-30

Fig. 17. Visual comparison of the sample data in the Amazon basin $\left(2-4^{\circ} \mathrm{S}, 68-71^{\circ} \mathrm{W}\right)$. 


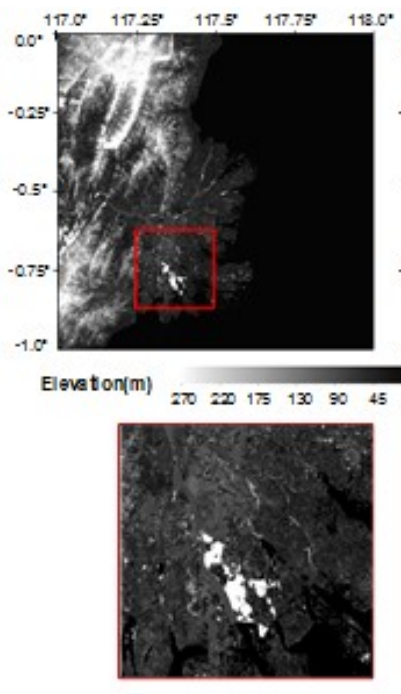

(a) ASTER GDEM

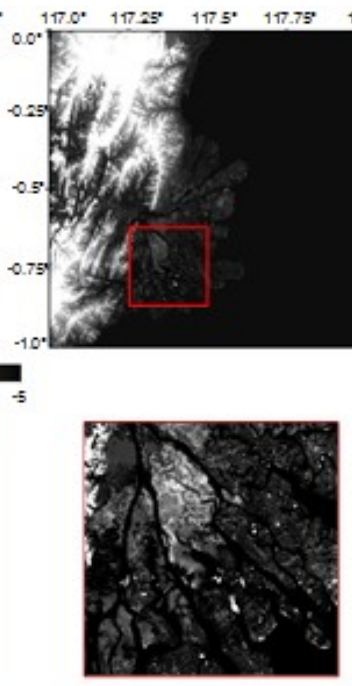

(b) AW3D30
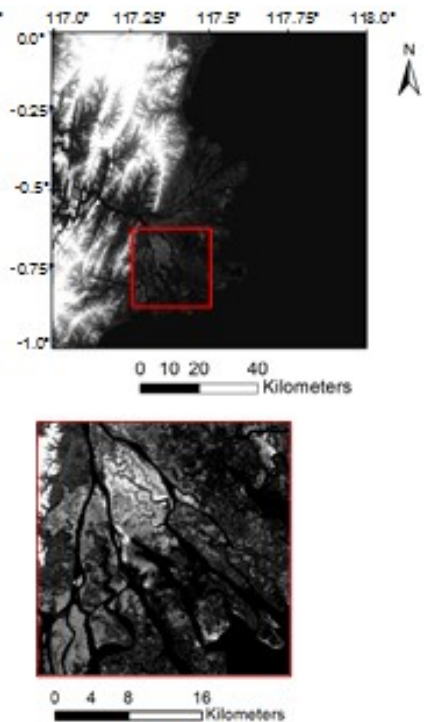

(c) GSDEM-30

Fig. 18. Visual comparison of the sample data in a coastline area $\left(0-1^{\circ} \mathrm{S}, 117-118^{\circ} \mathrm{N}\right)$.

\subsection{High-latitude data analysis}

The high-latitude data, referring to the data distributed between $60-82^{\circ} \mathrm{N}$ and $60-82^{\circ} \mathrm{S}$, are analyzed in this section. The SRTM data only cover the land areas between $60^{\circ} \mathrm{S}$ and $60^{\circ} \mathrm{N}$. With the release of the ASTER GDEM and AW3D DEM, the spatial coverage of the mid- and high-resolution elevation data has been extended to the high-latitude areas. Most of the SRTM-derived products merge the elevation information from these two datasets to fill the vacant areas in the high latitudes. Thus, we focus on the comparison of the two datasets in this section.

In Fig. 19, the spatial coverage of the ASTER GDEM v2 and AW3D30 DEM v1.1 over the high-latitude regions is shown. In the figure, the white parts indicate the regions without data tiles, while the black pixels are the voids existing in the tiles. In comparison, it can be seen that there are more no-data areas and voids in the AW3D30 DEM. Based on the statistics, for the northern part of the globe, there are 4808 data tiles between $60^{\circ}$ and $82^{\circ} \mathrm{N}$ for the ASTER GDEM, while there are only 4407 for the AW3D DEM. Although the spatial coverage of the ASTER GDEM is wider than that of the AW3D DEM, the data quality shows the opposite trend. There are clearly many more data anomalies existing in the GDEM tiles, based on a visual examination, which seriously affects the practical use of the elevation information. More details can be found in Table 3, where the total numbers of data tiles and the numbers of tiles with voids (value: -9999) and spurious artefacts are given. Considering the data quality, the GSDEM-30 use the elevation of AW3D for the high-latitude areas.

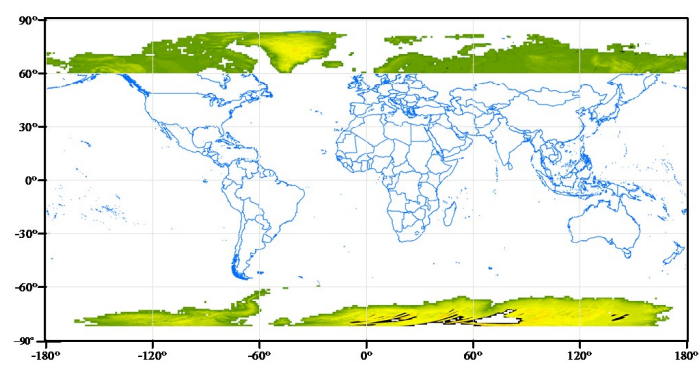

(a) ASTER GDEM

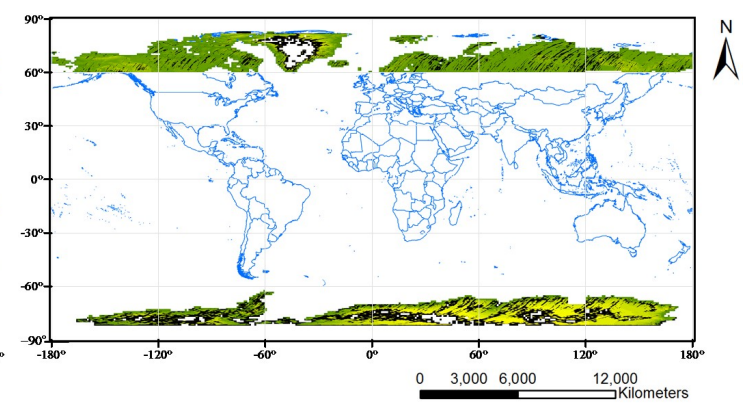

(b) AW3D30

Fig. 19 The spatial coverage of the ASTER and AW3D30 DEMs over high-latitude regions. 
Table 3 The statistics of the data distribution for the high-latitude regions.

\begin{tabular}{|c|c|c|c|c|c|c|}
\hline \multirow{2}{*}{ Dutaset } & \multicolumn{3}{|c|}{$60^{\circ}-82^{\circ} N$} & \multicolumn{3}{|c|}{$60^{\circ}-82^{\circ} S$} \\
\hline & Total & With voids & With artefacts & Total & With voids & With artefacts \\
\hline ASTER GDEM & 4808 & 1745 & 4626 & 3532 & 452 & 3381 \\
\hline AW3D30 & 4407 & 3824 & 3612 & 3529 & 2651 & 1293 \\
\hline
\end{tabular}

\section{Conclusions}

In this paper, we have introduced a global seamless DEM based on multi-source data fusion, which we refer to as GSDEM-30. Meanwhile, we have tried to present a comprehensive evaluation of GSDEM-30. In addition to GSDEM-30, the representative DEM datasets were also analyzed in our work, i.e., the ASTER GDEM, the AW3D30 DEM.

According to the results, GSDEM-30 is of good quality overall combining the respective advantages of the SRTM1, AW3D30, ASTER GDEM, and ICESat GLAS data. However, we also find that "no dataset is perfect" [묘]. Although data fusion is an effective way to fill the voids and suppress the artefacts in a single dataset, the uncertainties should be investigated before using the fused datasets. For example, the effectiveness of the point-surface fusion for GSDEM-30 might be limited to the distribution of GLAS tracks. Moreover, the water mask extracted from the remote sensing images for the fine editing of data tiles, might lead to inaccurate water boundary due to the classification errors. In addition, due to the inaccurate observations of the auxiliary data, there are still some artefacts in the void-filled version GSDEM-30. Therefore, for the applications with different demands of data resolution, and in different regions, different data can be selected based on the data quality. The accuracy validation results given in this paper will help the user to evaluate the data quality.

In addition to the voids and artefacts, there are relatively fewer works investigating other major error components of elevation data, such as the absolute bias and the tree height bias. The team behind the processing of MERIT DEM has conducted a significant work. However, due to the sparse track of ICESat GLAS data all over the world, the performance of the global absolute bias and tree height bias correction can vary from region to region. Therefore, we did not consider the vegetation height removal in the current version of GSDEM-30. In our future work, advanced editing of GSDEM-30 is expected, with more precise global correction of multiple biases.

Author Contributions: Conceptualization, Linwei Yue and Huanfeng Shen; methodology, Linwei Yue; validation, Linwei Yue, and Lu Liu; formal analysis, Linwei Yue and Lu Liu; investigation, Linwei Yue and Huanfeng Shen; writing - original draft preparation, Liniwei Yue and Lu Liu; writing-review and editing, Linwei Yue, Huanfeng Shen, Qiangqiang Yuan and Liangpei Zhang; supervision, Huanfeng Shen and Liangpei Zhang.

Funding: This research was funded by National Natural Science Foundation of China (41801263), Open Fund of Key Laboratory of Geographic Information Science (Ministry of Education), East China Normal University (Grant No. KLGIS2017A03) and the Fundamental Research Funds for the Central Universities, China University of Geosciences (Wuhan) (CUG170663).

Conflicts of Interest: The authors declare no conflict of interest. 


\section{References}

1. Yang, L.; Meng, X.; Zhang, X. SRTM DEM and its application advances. International Journal of Remote Sensing 2011, 32, 3875-3896.

2. Berry, P.A.M.; Garlick, J.D.; Smith, R.G. Near-global validation of the SRTM DEM using satellite radar altimetry. Remote Sensing of Environment 2007, 106, 17-27.

3. Tachikawa, T.; Hato, M.; Kaku, M.; Iwasaki, A. Characteristics of ASTER GDEM version 2. In Proceedings of Geoscience and Remote Sensing Symposium; pp. 3657-3660.

4. Tadono, T.; Takaku, J.; Tsutsui, K.; Oda, F.; Nagai, H. Status of “ALOS World 3D (AW3D)” global DSM generation. In Proceedings of Geoscience and Remote Sensing Symposium; pp. 3822-3825.

5. Rizzoli, P.; Martone, M.; Gonzalez, C.; Wecklich, C.; Borla Tridon, D.; Bräutigam, B.; Bachmann, M.; Schulze, D.; Fritz, T.; Huber, M. Generation and performance assessment of the global TanDEM-X digital elevation model. Isprs Journal of Photogrammetry \& Remote Sensing 2017, 132, 119-139.

6. Fisher, P.F.; Tate, N.J. Causes and consequences of error in digital elevation models. Progress in Physical Geography 2006, 30, 467-489.

7. Hirano, A.; Welch, R.; Lang, H. Mapping from ASTER stereo image data: DEM validation and accuracy assessment. ISPRS Journal of Photogrammetry \& Remote Sensing 2003, 57, 356-370.

8. Toutin, T. Impact of terrain slope and aspect on radargrammetric DEM accuracy. ISPRS Journal of Photogrammetry \& Remote Sensing 2003, 57, 228-240.

9. Noh, M.-J.; Howat, I.M. Automated stereo-photogrammetric DEM generation at high latitudes: Surface Extraction with TIN-based Search-space Minimization (SETSM) validation and demonstration over glaciated regions. GIScience \& Remote Sensing 2015, 52, 198-217.

10. Gao, X.; Liu, Y.; Li, T.; Wu, D. High Precision DEM Generation Algorithm Based on InSAR Multi-Look Iteration. Remote Sensing 2017, 9, 741.

11. Frey, H.; Paul, F. On the suitability of the SRTM DEM and ASTER GDEM for the compilation of topographic parameters in glacier inventories. International Journal of Applied Earth Observation $\mathcal{E}$ Geoinformation 2012, 18, 480-490.

12. Krieger, G.; Moreira, A.; Fiedler, H.; Hajnsek, I.; Werner, M.; Younis, M.; Zink, M. TanDEM-X: A Satellite Formation for High-Resolution SAR Interferometry. IEEE Transactions on Geoscience and Remote Sensing 2007, 45, 3317-3341, doi:10.1109/TGRS.2007.900693.

13. Rizzoli, P.; Martone, M.; Gonzalez, C.; Wecklich, C.; Borla Tridon, D.; Bräutigam, B.; Bachmann, M.; Schulze, D.; Fritz, T.; Huber, M., et al. Generation and performance assessment of the global TanDEM-X digital elevation model. ISPRS Journal of Photogrammetry and Remote Sensing 2017, 132, 119-139, doi:https://doi.org/10.1016/j.isprsjprs.2017.08.008.

14. Reuter, H.I.; Nelson, A.; Jarvis, A. An evaluation of void-filling interpolation methods for SRTM data. International Journal of Geographical Information Science 2007, 21, 983-1008.

15. Gallant, J. Adaptive smoothing for noisy DEMs. Geomorphometry 2011 2011, 7-9.

16. Baier, G.; Rossi, C.; Lachaise, M.; Zhu, X.X.; Bamler, R. A Nonlocal InSAR Filter for High-Resolution DEM Generation From TanDEM-X Interferograms. IEEE Transactions on Geoscience and Remote Sensing 2018, 1-15.

17. Tarekegn, T.H.; Sayama, T. Correction of SRTM DEM artefacts by Fourier transform for flood inundation modeling. Journal of Japan Society of Civil Engineers, Ser. B1 (Hydraulic Engineering) 2013, 69, I_193-I_198.

18. Takaku, J.; Iwasaki, A.; Tadono, T. Adaptive filter for improving quality of ALOS PRISM DSM. In Proceedings of 2016 IEEE International Geoscience and Remote Sensing Symposium (IGARSS); pp. 5370-5373.

19. Robinson, N.; Regetz, J.; Guralnick, R.P. EarthEnv-DEM90: A nearly-global, void-free, multi-scale smoothed, 90m digital elevation model from fused ASTER and SRTM data. ISPRS Journal of Photogrammetry E Remote Sensing 2014, 87, 57-67.

20. Rengarajan, R.; Sampath, A.; Storey, J.; Choate, M. Validation of geometric accuracy of global land survey (GLS) 2000 data. Photogrammetric Engineering E Remote Sensing 2015, 81, 131-141.

21. Yamazaki, D.; Ikeshima, D.; Tawatari, R.; Yamaguchi, T.; O'Loughlin, F.; Neal, J.C.; Sampson, C.C.; Kanae, S.; Bates, P.D. A high-accuracy map of global terrain elevations. Geophysical Research Letters 2017, 44.

22. Yue, L.; Shen, H.; Zhang, L.; Zheng, X.; Zhang, F.; Yuan, Q. High-quality seamless DEM generation blending SRTM-1, ASTER GDEM v2 and ICESat/GLAS observations. Isprs Journal of Photogrammetry $\mathcal{E}$ Remote Sensing 2017, 123, 20-34. 
23. Amatulli, G.; Domisch, S.; Tuanmu, M.N.; Parmentier, B.; Ranipeta, A.; Malczyk, J.; Jetz, W. A suite of global, cross-scale topographic variables for environmental and biodiversity modeling. Scientific Data 2018, 5,180040

24. Arefi, H.; Reinartz, P. Accuracy enhancement of ASTER global digital elevation models using ICESat data. Remote Sensing 2011, 3, 1323-1343.

25. Jarvis, A.; Reuter, H.I.; Nelson, A.; Guevara, E. Hole-filled seamless SRTM data V4. International Centre for Tropical Agriculture (CIAT) 2008.

26. Yue, L.; Shen, H.; Yuan, Q.; Zhang, L. Fusion of multi-scale DEMs using a regularized super-resolution method. International Journal of Geographical Information Science 2015, 29, 2095-2120.

27. Hirt, C. Artefact detection in global digital elevation models (DEMs): The Maximum Slope Approach and its application for complete screening of the SRTM v4.1 and MERIT DEMs. Remote Sensing of Environment 2018, 207, 27-41.

28. Rosen, P.; Eineder, M.; Rabus, B.; Gurrola, E. SRTM-Mission - cross comparison of X and C band data properties. In Proceedings of Geoscience and Remote Sensing Symposium, 2001. IGARSS '01. IEEE 2001 International; pp. 751-753 vol.752.

29. Minowa, M.; Sugiyama, S.; Sakakibara, D.; Sawagaki, T. Contrasting glacier variations of Glaciar Perito Moreno and Glaciar Ameghino, Southern Patagonia Icefield. Annals of Glaciology 2015, 56, $26-32$.

30. Mukherjee; Samadrita; Sandip; Ghosh; Aniruddha; Garg; R., D.; Mukhopadhyay. Evaluation of vertical accuracy of open source Digital Elevation Model;(DEM). International Journal of Applied Earth Observation $\mathcal{E}$ Geoinformation 2013, 21, 205-217.

31. Michael Kobrick, R.C. NASA Shuttle Radar Topography Mission Global 1 arc second. NASA EOSDIS Land Processes DAAC: 2013; 10.5067/MEaSUREs/SRTM/SRTMGL1.003.

32. Buckley, S. NASADEM: Creating a New NASA Digital Elevation Model and Associated Products. Availabe online:

https://earthdata.nasa.gov/earth-science-data-systems-program/competitive-programs/measures/nasadem (accessed on May 3).

33. Tachikawa, T.; Kaku, M.; Iwasaki, A.; Gesch, D.B.; Oimoen, M.J.; Zhang, Z.; Danielson, J.; Krieger, T.; Curtis, B.; Haase, J. ASTER Global Digital Elevation Model Version 2 - Summary of validation results. Kim Fakultas Sastra Dan Budaya 2011.

34. Gesch, D.; Oimoen, M.; Danielson, J.; Meyer, D. Validation of the Aster Global Digital Elevation Model Version 3 Over the Conterminous United States. In Proceedings of ISPRS - International Archives of the Photogrammetry, Remote Sensing and Spatial Information Sciences, Prague, Crech Republic, 12-19 July; pp. 143-148.

35. Tadono, T.; Ishida, H.; Oda, F.; Naito, S.; Minakawa, K.; Iwamoto, H. Precise Global DEM Generation by ALOS PRISM. 2014, ii-4, 71-76.

36. Takaku, J.; Tadono, T.; Tsutsui, K.; Ichikawa, M. Validation of "AW3D" Global Dsm Generated from Alos Prism. ISPRS Annals of Photogrammetry Remote Sensing \& Spatial Informa 2016, III-4, 25-31.

37. Takaku, J.; Tadono, T.; Tsutsui, K.; Ichikawa, M. Quality Improvements of 'AW3D'Global Dsm Derived from Alos Prism. In Proceedings of IGARSS 2018-2018 IEEE International Geoscience and Remote Sensing Symposium; pp. 1612-1615.

38. JAXA. Availabe online: https://www.eorc.jaxa.jp/ALOS/en/aw3d30/index.htm (accessed on April).

39. Bonnet, M.P.; Timouk, F.; Molina, J.; Lavado-Casimiro, W.; Arsen, A.; Garnier, J. Accuracy assessment of SRTM v4 and ASTER GDEM v2 over the Altiplano watershed using ICESat/GLAS data. International Journal of Remote Sensing 2015, 36, 465-488.

40. Abshire, J.B.; Sun, X.; Riris, H.; Sirota, J.M.; McGarry, J.F.; Palm, S.; Yi, D.; Liiva, P. Geoscience laser altimeter system (GLAS) on the ICESat mission: on-orbit measurement performance. Geophysical Research Letters 2005, 32.

41. Bhang, K.J.; Schwartz, F.W.; Braun, A. Verification of the Vertical Error in C-Band SRTM DEM Using ICESat and Landsat-7, Otter Tail County, MN. IEEE Transactions on Geoscience E Remote Sensing 2007, 45, 36-44.

42. Wang, X.; Cheng, X.; Gong, P.; Huang, H.; Li, Z.; Li, X. Earth science applications of ICESat/GLAS: a review. International Journal of Remote Sensing 2011, 32, 8837-8864.

43. Zhang, G.; Xie, H.; Kang, S.; Yi, D.; Ackley, S.F. Monitoring lake level changes on the Tibetan Plateau using ICESat altimetry data (2003-2009). Remote Sensing of Environment 2011, 115, 1733-1742. 
44. Huber, M.; Wessel, B.; Kosmann, D.; Felbier, A.; Schwieger, V.; Habermeyer, M.; Wendleder, A.; Roth, A. Ensuring globally the TanDEM-X height accuracy: Analysis of the reference data sets ICESat, SRTM and KGPS-tracks. In Proceedings of Geoscience and Remote Sensing Symposium,2009 IEEE International,igarss; pp. II-769-II-772.

45. Harding, D.J.; Gesch, D.B.; Carabajal, C.C.; Luthcke, S.B. Application of the shuttle laser altimeter in an accuracy assessment of GTOPO30, a global 1-kilometer digital elevation model. International Archives of Photogrammetry and Remote Sensing 1999, 32, 81-85.

46. Danielson, J.J.; Gesch, D.B. Global multi-resolution terrain elevation data 2010 (GMTED2010); 2331-1258; US Geological Survey: 2011.

47. de Ferranti, J. Availabe online: http://www.viewfinderpanoramas.org/index.htm (accessed on 26, May).

48. Li, T.; Shen, H.; Yuan, Q.; Zhang, X.; Zhang, L. Estimating ground-level PM2. 5 by fusing satellite and station observations: A geo-intelligent deep learning approach. Geophysical Research Letters 2017, 44.

49. Luedeling, E.; Siebert, S.; Buerkert, A. Filling the voids in the SRTM elevation model - A TIN-based delta surface approach. ISPRS Journal of Photogrammetry and Remote Sensing 2007, 62, 283-294, doi:http://dx.doi.org/10.1016/j.isprsjprs.2007.05.004.

50. Pan, R.; Reeves, S.J. Efficient Huber-Markov edge-preserving image restoration. IEEE Transactions on Image Processing 2006, 15, 3728-3735.

51. Feng, M.; Sexton, J.O.; Channan, S.; Townshend, J.R. A global, high-resolution (30-m) inland water body dataset for 2000: First results of a topographic-spectral classification algorithm. International Journal of Digital Earth 2016, 9, 113-133.

52. Greenlee, S.; Oimoen, M.; Gesch, D. The National Elevation Dataset. Photogrammetric Engineering \& Remote Sensing 2002, 68, págs. 5-32.

53. Ban, Y.; Li, S. China: Open access to Earth land-cover map. Nature 2015, 514, 434.

54. Xie, H.; Tong, X.; Meng, W.; Liang, D.; Wang, Z.; Shi, W. A Multilevel Stratified Spatial Sampling Approach for the Quality Assessment of Remote-Sensing-Derived Products. IEEE Journal of Selected Topics in Applied Earth Observations \& Remote Sensing 2016, 8, 4699-4713.

55. Grohmann, C.H. Evaluation of TanDEM-X DEMs on selected Brazilian sites: Comparison with SRTM, ASTER GDEM and ALOS AW3D30. Remote Sensing of Environment 2018, 212, 121-133.

56. Carabajal, C.C.; Harding, D.J. ICESat validation of SRTM C-band digital elevation models. Geophysical Research Letters 2005, 32.

57. Su, Y.; Guo, Q. A practical method for SRTM DEM correction over vegetated mountain areas. ISPRS Journal of Photogrammetry and Remote Sensing 2014, 87, 216-228, doi:http://dx.doi.org/10.1016/j.isprsjprs.2013.11.009.

58. Giles, J.R.A.; Marsh, S.H.; Napier, B. Dataset acquisition to support geoscience. Geological Society London Special Publications 2010, 345, 135-143. 